\title{
EEG and Eye Tracking in Attention Paid to Charity Advertising: An Abstract
}

\author{
Manuel Alonso Dos Santos, Bryan Gárate, Mariela Jara, and Axiel Saavedra
}

\begin{abstract}
Our aim is to analyze the impact of gender and image valence on the attention paid and the intention to make a donation in nongovernmental charity advertising. The experiment was carried out with intra-subject (logo, text, image) and inter-subject (valence, gender) factors. The data are collected using an electroencephalogram and the eye-tracking technique from three areas of interest: image, text, and logo. More attention was found to be paid to images than to the other areas of interest (EEG, number of fixations, and average time of fixation). In particular, negative images got higher attention levels. No differences were found with regard to gender and the intention to donate in terms of attention paid to the advertisements. However, women proved to be more willing to make a donation. Extrapolation of results requires caution, as the sample is not representative. This is an exploratory research. The neurophysiological tools employed also require further research for the validation of results. Different strategies are put forward in this study for increasing attention rates, such as the use of images depicting people, a visible face, and a negative impact. In this way, NGOs will be able to increase their visibility and advertising efficiency. To our knowledge, attention has not yet been measured combining two neurophysiological techniques in charity advertising. The image valence and its impact on the attention paid to charity advertisements are addressed from a new angle, while some contradictions found in literature are resolved.
\end{abstract}

M.A.D. Santos $(\bowtie) \bullet$ B. Gárate $\bullet$ M. Jara $\bullet$ A. Saavedra

Universidad Católica de la Santísima Concepción, Concepción, Chile

e-mail:malonso@ucsc.cl; bgarate@icomercial.ucsc.cl; mjara@icomercial.ucsc.cl;

asaavedra@icomercial.ucsc.cl 